Bulletin of JSME, Vol. 29, No. 252 June 1986

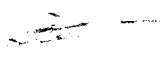

Paper No. $85-0215$

A Study on the Axially Curved Mixed-Flow Vaneless Diffusers*

By Takashi AWAI**, Tomohiro NAKAGAWA***

and Toshimichi SAKAI****

\begin{abstract}
From a view point of machine dimension, the axially curved mixed-flow diffuser has a large benefit compared with the purely conical or the raaially curved mixed-flow diffuser. In the present study, the flow pattern perimental recovery in the axially curved mixed-flow diffusers are exly conical diffusers. ly conical diffusers. The pressure recovery factor and diffuser effecconical diffuser axially curved diffuser were low compared with the purely conical diffuser, but the difference between them was small. By use of rectified and a the inner wall, the in the axily curved diffuser was rectifled and a favorable performance was obtained.
\end{abstract} Key Words: Fluid Machine Element, Boundary Layer, Turbomachine, Centrifugal
Compressor, Diffuser

1. Introduction

The flow pattern and performance of axially curved diffusers for mixed-flow turbomachines are examined. In the mixedflow turbomachine, the purely conical or the radially curved annular diffuser is generally used for the purpose of recovery of static pressure. However the outer diameters of these machines are almost the same as those of centrifugal machines. In the axially curved mixed-flow diffusers, it is possible to decrease the outer diem eter of machines and it brings advantages for practical use. But in the axially curved diffusers, the static pressure near the inner convex wall tends to become much lower than that near the outer concave lower than that near the outer concave wall due to the existence of a body force normal to the diffuser walls resulting from the radius of curvature of diffuser at the meridional section and flow swirl. Together with the development of a boundary layer near the inner convex a boundlow energy region begins to form near the inner wall. This will bring down the the chine performance. In this study, the axially curved mixed-flow diffusers are examined and experimental flow pattern and the performance are compared with those of the purely conical annular diffuser(Type
PC).

* Received 1st March, 1985.

* Graduate student, Department of Mechanical Engineering, The Science University of Tokyo, 1-3, Kagurazaka, Shinjuku-ku, Tokyo, 162, Japan.

* * Undergraduate student, Department of Mechanical Engineering, The Science University of Tokyo.

*** Professor, Department of Mechanical Engineering, The Science University of Tokyo.
2. Apparatus and Instrumentation

The experimental arrangement is shown in Fig.1. The air enters the diffuser at a predetermined velocity through a rotating screen which imparts a swirl to the flow at the diffuser inlet. For the purpose of removing the boundary layer at the inlet, the walls are sharpened to knife edges. Total and static pressures and the flow direction are measured by use of calibrated three-hole cobra type probes which are made of $0.7 \mathrm{~mm}$ stainless steel tubes. One to three fold screens are fitted at the diffuser outlet to provide a resistance to the out flow. The axial symmetry of the flow in the diffuser is confirmed by normal directional integration of the meridional component of velocity. The following four types of diffusers are used in this study:

Type A: A diffuser having the same diffusion ratio as the purely conical parallel walled diffuser.

Type B: A diffuser having constant width. Radius of curvature of this diffuser is equal to $120 \mathrm{~mm}$ at the outer wall.

Type E: A diffuser having constant sectional area. Radius of curvature of this diffuser is equal to $120 \mathrm{~mm}$ at the outer wall.

Type D: A diffuser having guide fences on the inner wall of Type A diffuser: a set of twelve guide fences $1 \mathrm{~mm}$ thick and $5 \mathrm{~mm}$ high; the camber line has a logarithmic spiral with an angle $\theta=30$ degrees.

Table 1 shows dimensions of those diffusers.

Fig.2 shows the area ratio against the distance which is measured from diffuser inlet to the downstream-direction. 


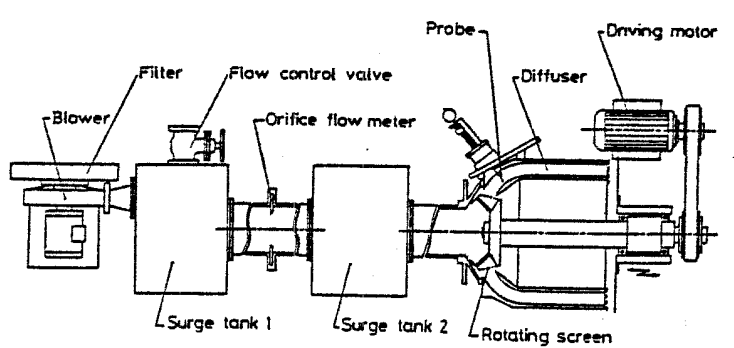

Fig. 1 Arrangement of test rig

Table 1 Dimensions of diffusers

\begin{tabular}{|l|c|c|c|c|}
\hline \multicolumn{1}{|c|}{ Diffuser Type } & A, D & B & E & PC \\
\hline $\begin{array}{l}\text { Depth of } \\
\text { Diffuser Inlet bo }\end{array}$ & 26 & 33 & 33 & 26 \\
\hline $\begin{array}{l}\text { Radius of } \\
\text { Diffuser Inlet }\end{array}$ O & 100 & 101.75 & 101.75 & 104.35 \\
\hline $\begin{array}{l}\text { Half of Inlet } \\
\text { Cone Angle }\end{array}$ & $60^{\circ}$ & $60^{\circ}$ & $68.4^{\circ}$ & $60^{\circ}$ \\
\hline $\begin{array}{l}\text { Depth of } \\
\text { Axial Flow Section }\end{array}$ & 33 & 33 & 19.75 & 26 \\
\hline Radius Ratio & 1.64 & 1.61 & 1.67 & 1.8 \\
\hline $\begin{array}{l}\text { Outer Diameter of } \\
\text { Diffuser }\end{array}$ & 376 & 376 & 376 & 500 \\
\hline
\end{tabular}

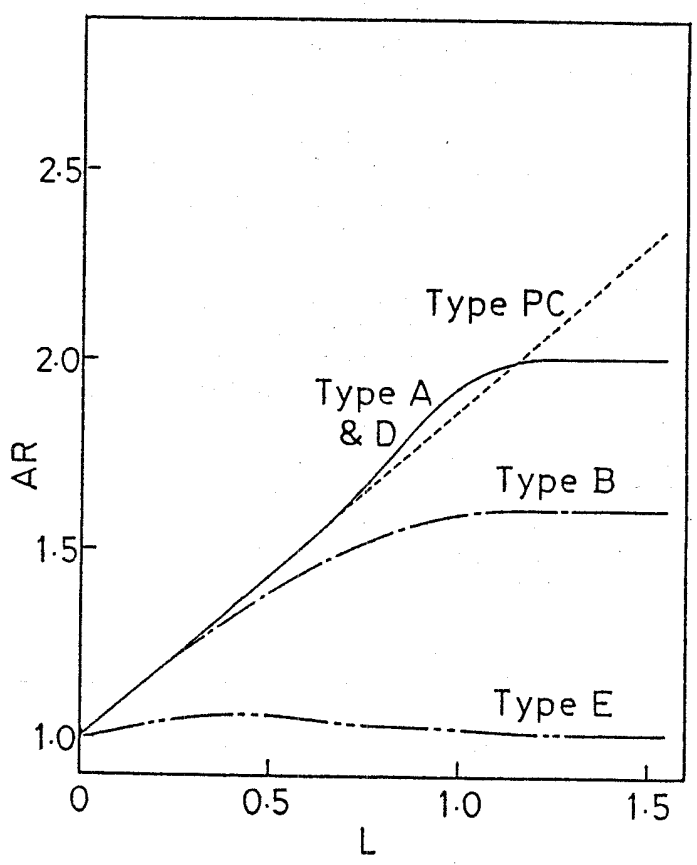

Fig. 2 Area ratio vs. distance from diffuser inlet

Experiments are performed at Reynolds numbers of $2.0 \times 10^{5} \sim 2.1 \times 10^{5}$ and the characteristic length and velocity are the diffuser inlet diameter and mean velocity of the flow at the diffuser inlet.

\section{Numerical Analysis}

Assuming that the flow is steady and inviscid, the gradient of meridional velocity component normal to the walls is $\frac{d C_{m}}{d n}=-\frac{C_{m}}{R_{s}}+\frac{1}{C_{m}} \cdot \frac{d h_{0}}{d n}-\frac{C_{0}}{C_{m}}\left(\frac{d C_{0}}{d n}+\frac{C_{0}}{r} \cos \alpha\right) \ldots \ldots . .(1)$

Where ho is total enthalpy, Rs is radius of curvature of the streamline at the ridional plane, $C_{\theta}$ is tangential the mecomponent, $r$ is $\theta$ is tangential velocity $\alpha$ is angle betheam surface. Eq. (1) is integrated with the assumption that the angular momentum is conserved along the streamlines and total enthalpy is constant on each streamline. Calculated values of inner flow and static pressure are compared with experimental values.

\section{Discussion of Results}

Fig. 3 shows typical streamlines and distribution of static pressure coefficients of the purely conical diffuser Type PC. The inlet flow model was approximately uniform normal to the diffuser walls and inlet flow angle $\theta i$ was equal to 30 degrees. In this case the static pressure gradient normal to the walls was considerable due to the existence of a body force due to swirl. Together with the radical development of the boundary layer near the inner wall, a low energy region began to form near the inner wall. For this reason flow reversal was liable to occur near the inner wall. In this study the inner flow and performance of the axially curved diffusers are compared with those of Type PC.
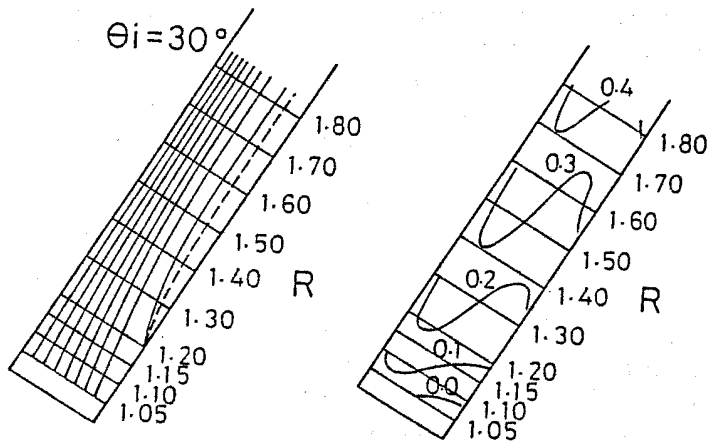

Fig. 3 A flow pattern and distribution of pressure recovery coefficients in Type PC diffuser

Fig. 4 and Fig.5 show the pattern of streamlines and meridional component of velocity in the axially curved diffusers Type A, Type B and Type E. The inlet flow models are approximately uniform normal to the walls and the inlet flow angle $\theta i$ is equal to 30 degrees. The streamlines in Type A and Type B gradually incline toward the outer wall to the downstream direction with the development of a boundary layer and flow reversal occur near the inner wall. Flow reversal is observed around radius ratio $R=1.2$ in Type $A$ and $a-$ round $R=1.3$ in Type $B$. But these flow reversals disappear at the downstream annular axial flow passage. The size of flow reversal region is influenced by the diffusion ratio. In Type $A$ it is larger than that in Type PC. The percentage occupied 
by flow reversal area to the diffuser flow passage is $18.3 \%$ in Type PC, $19.4 \%$ in Type $A$ and $13.5 \%$ in Type B. In Type $E$ diffuser the increment of static pressure is due to a decrease in tangential component of velocity so that the streamwise velocity gradient is small. A little development of the boundary layer is observed near the inner wall, but flow reversal is not observed and a favorable inner flow is obtained.
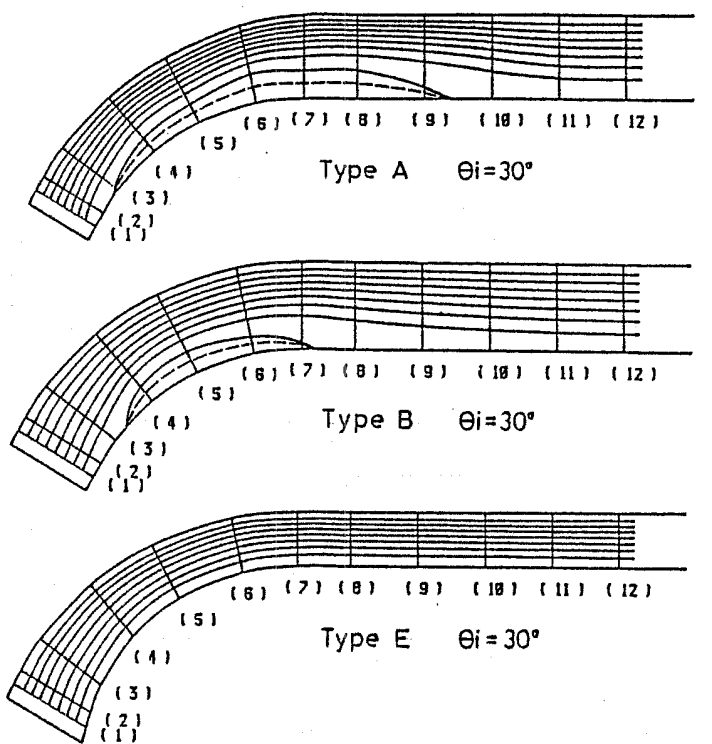

Fig. 4 Flow patterns of the examined diffusers

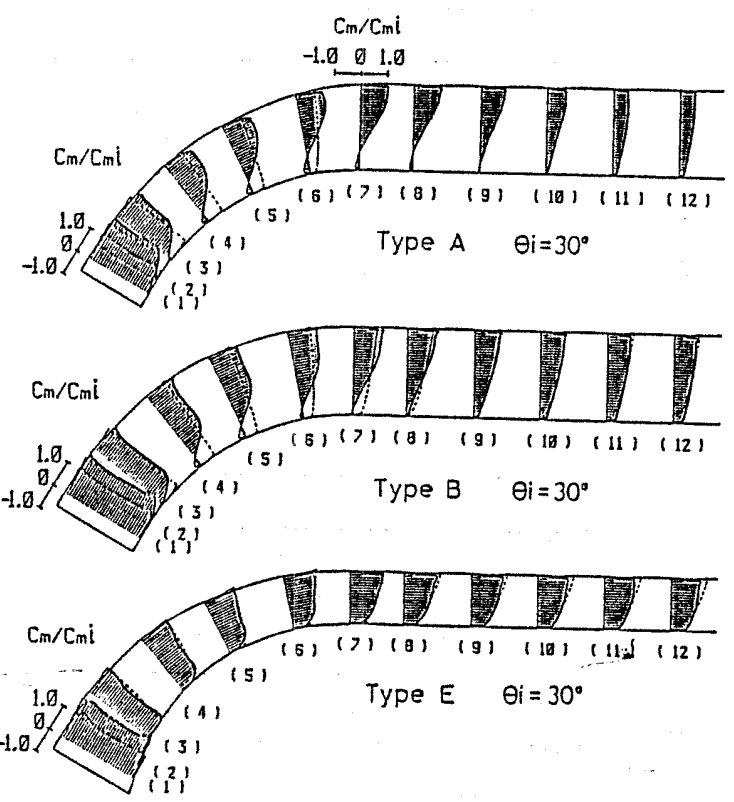

Fig. 5 Distribution of meridional velocity components

In Fig.5 broken lines show theoretical value for an inviscid flow evaluated by Eq. (1). In this experiment the inlet flow model has an enthalpy gradient normal to the walls so that the velocity near the outer wall is higher than that of inner wall. In the case of the favorable flow (Type E $\theta i=30,45$, Type B $\theta i=45,60$ and Type A $\theta i=60$ ), a difference between experimental and calculated values is small. Fig. 6 shows the measured tangential component of velocity at the sites No.4 and No.11. Broken lines show calculated values for an inviscid flow. In Type $A$ and Type $B, C e$ showes lower values compared with calculated values near the inner wall at the site No. 4 where flow reversal is observed. In Type $\mathrm{E}$ the angular momentum is approximately conserved. At the site No. 11 Ce showes a lower value compared with calculated value near the both walls on account of the skin friction at the walls.
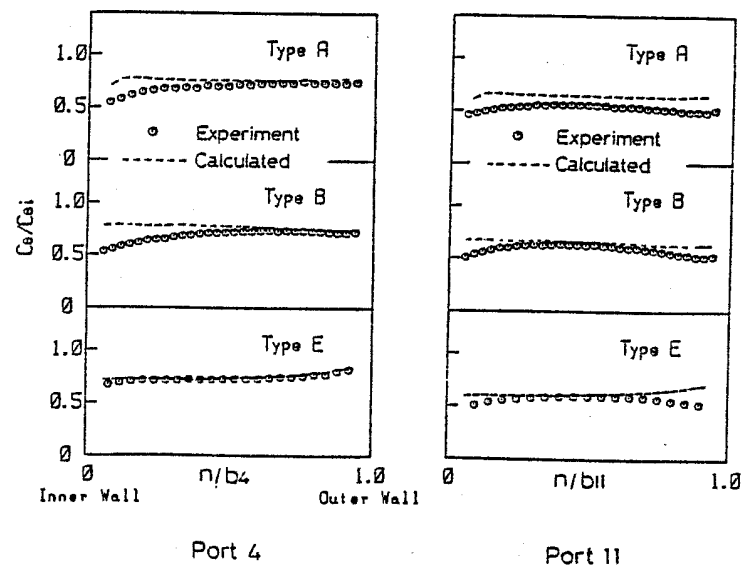

Fig. 6 Tangential velocity component

Fig.7 shows the total pressure coefficients Cpt, Cpt=(Pt-Psi)/(Pti-Psi) where Pt is total pressure at a given point, Psi is mass-flow-averaged inlet static pressure and Pti is mass-flow-averaged inlet total pressure. Fig. 8 shows the pressure recovery coefficients Cps, $\mathrm{Cps}=(\mathrm{Ps}-\mathrm{Psi}) /$ (Pti-Psi) where Ps is static pressure at a given point. Broken lines in Fig.8 show theoretical value for an inviscid flow evaluated by Eq.(1).
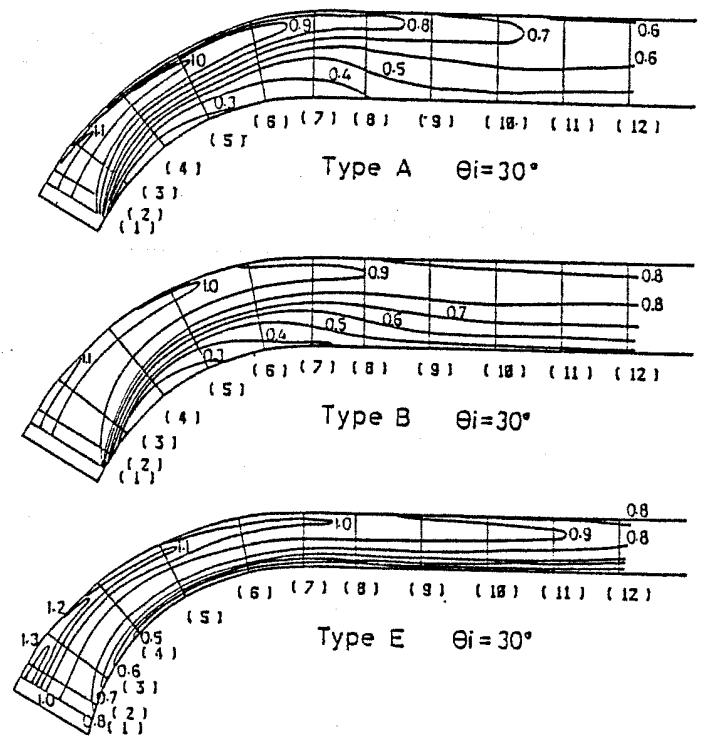

Fig. 7 Distribution of total pressure coefficients Cp 

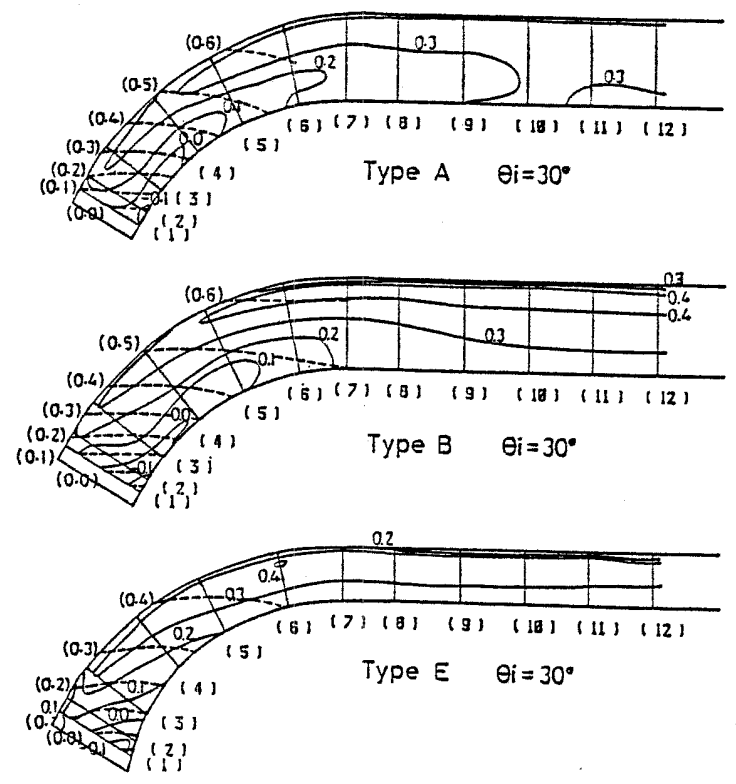

Fig. 8 Distribution of pressure recovery coefficients $\mathrm{Cps}$

In Fig.7 the low energy region, i.e. small Cpt, exists near the inner walls of Type $A$ and Type B. And in that region, Cps is fairly small compared with calculated value and static pressure gradient normal to the walls is larger than calculated value. In Type E static pressure recovery near the inner wall is favorable and static pressure gradient normal to the walls is smaller than those of Type A and Type B because development of the boundary layer near the inner wall is not so rapid.

Fig.9 shows streamlines and distribution of static pressure coefficients of Type D. The inlet flow condition of Type D is similar to that of Type A. From this figure it is seen that the flow near the inner wall is much rectified compared with that of Type A. Development of boundary layer is recognised at the downstream of the guide fences, but flow reversal is not observed. Static pressure recovery is favorable and higher diffuser performance is obtained.
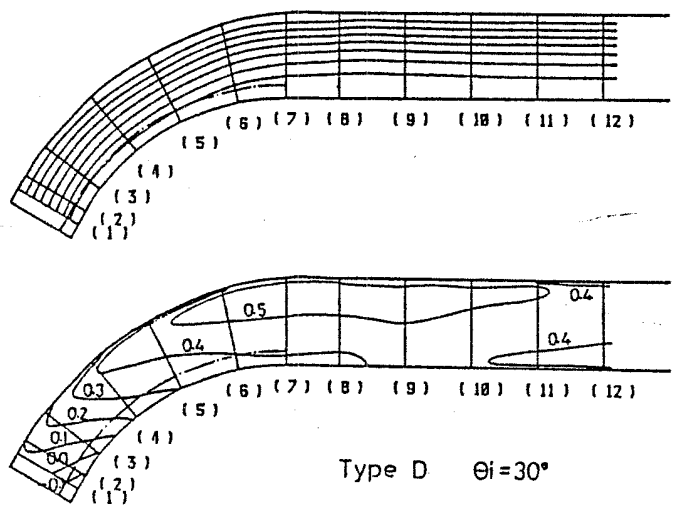

Fig. 9 Flow patterns and distribution of pressure recovery coefficients in Type D diffuser
In Fig.10 a drop in the total pressure and static pressure recovery of curved diffusers Type A, Type D, Type B and Type $E$ are shown. In this figure the mass-flow-averaged values in the normal direction are plotted along the meridional axis, and the variations in the static and the total pressure are normalized by means of the dynamic pressure measured at the diffuser inlet. The broken lines show the static pressure recovery for an inviscid flow. From this figure the correlation between the inner flow and performance is clearly seen. In the region where boundary layer developed, the static pressure recovery is not favorable and the drop in the total pressure is remarkable. In Type $A$ and Type $B$ when $\theta i$ is equal to $45 \mathrm{de}-$ grees, pressure recovery is large compared with the cases when $\theta i$ is equal to $30 \mathrm{de}-$ grees because of small boundary layer development due to short length of stream-
lines.
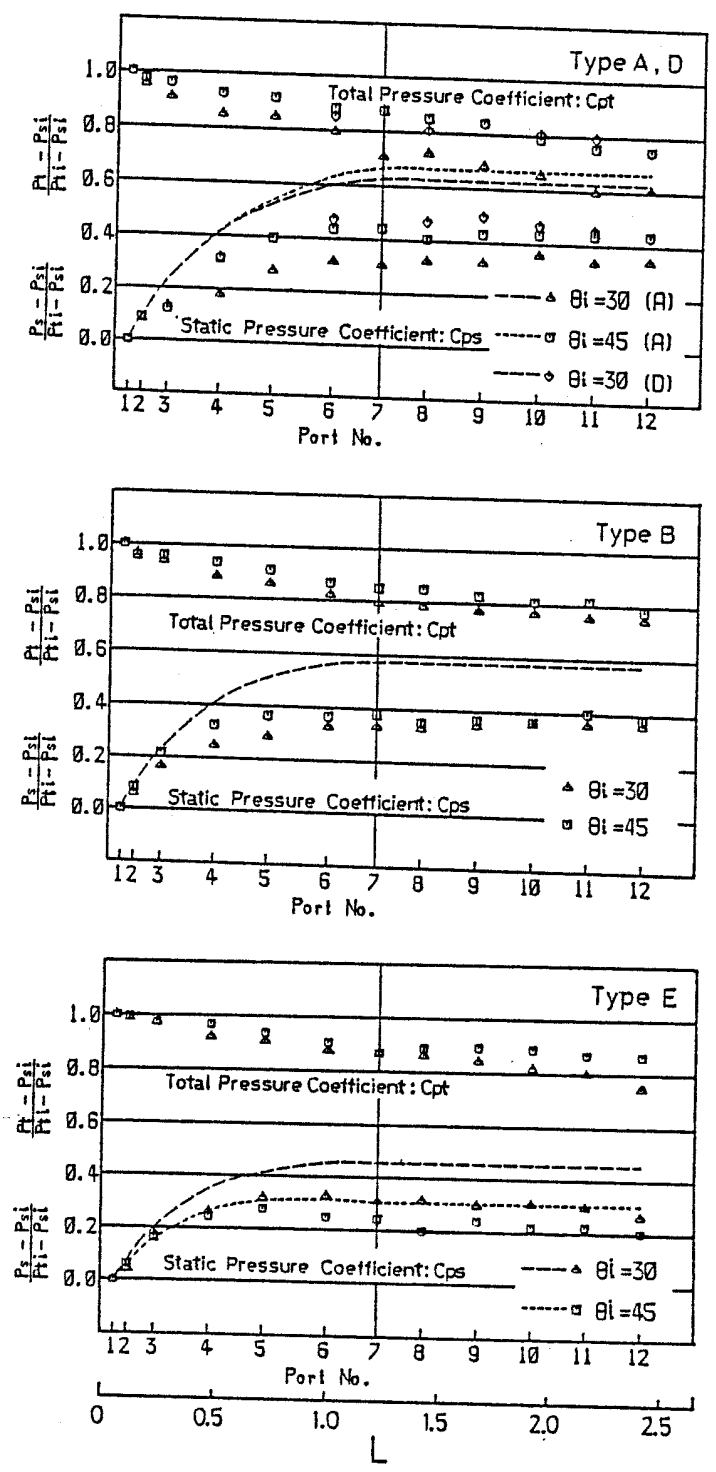

Fig. 10 Total pressure coefficient Cpt and pressure recovery coefficient Cps in axially curved diffuser 
Table 2 The pressure recovery coefficient $\mathrm{Cps}$ and diffuser effectiveness $\eta_{\mathrm{p}}$

\begin{tabular}{|c|c|c|c|c|c|c|c|l|l|l|l|l|}
\hline Diffuser Type & \multicolumn{2}{|c|}{ PC } & \multicolumn{3}{|c|}{ A } & \multicolumn{3}{c|}{ B } & D & \multicolumn{3}{c|}{ E } \\
\hline Inlet Flow Angle & $25^{\circ *}$ & $30^{\circ}$ & $30^{\circ}$ & $45^{\circ}$ & $60^{\circ}$ & $30^{\circ}$ & $45^{\circ}$ & $60^{\circ}$ & $30^{\circ}$ & $20^{\circ}$ & $30^{\circ}$ & $45^{\circ}$ \\
\hline Back Flow Region & YES* & YES & YES & YES & NO & YES & NO & NO & NO & YES & NO & NO \\
\hline CPSth & 0.66 & 0.66 & 0.60 & 0.63 & 0.66 & 0.56 & 0.56 & 0.56 & 0.60 & 0.53 & 0.45 & 0.31 \\
\hline CPS & 0.39 & 0.39 & 0.32 & 0.44 & 0.46 & 0.33 & 0.37 & 0.42 & 0.47 & 0.40 & 0.33 & 0.25 \\
\hline$\eta_{P}$ & 0.59 & 0.59 & 0.52 & 0.69 & 0.69 & 0.59 & 0.66 & 0.74 & 0.79 & 0.76 & 0.73 & 0.79 \\
\hline
\end{tabular}

(*: with guide fence, back flow observed after guide fences)

Fig.11 shows a drop in the total pressure and static pressure recovery, and they are cornpared between purely conical diffuser Type PC and curved diffuser Type A when $\theta i$ is equal to 30 degrees. In Type A the drop in the total pressure is large and static pressure recovery is slow in the region from the site No.3 to No.4 because the size of flow reversal region is large. The pressure recovery in curved diffuser Type $A$ is lower than that in purely conical diffuser Type PC.

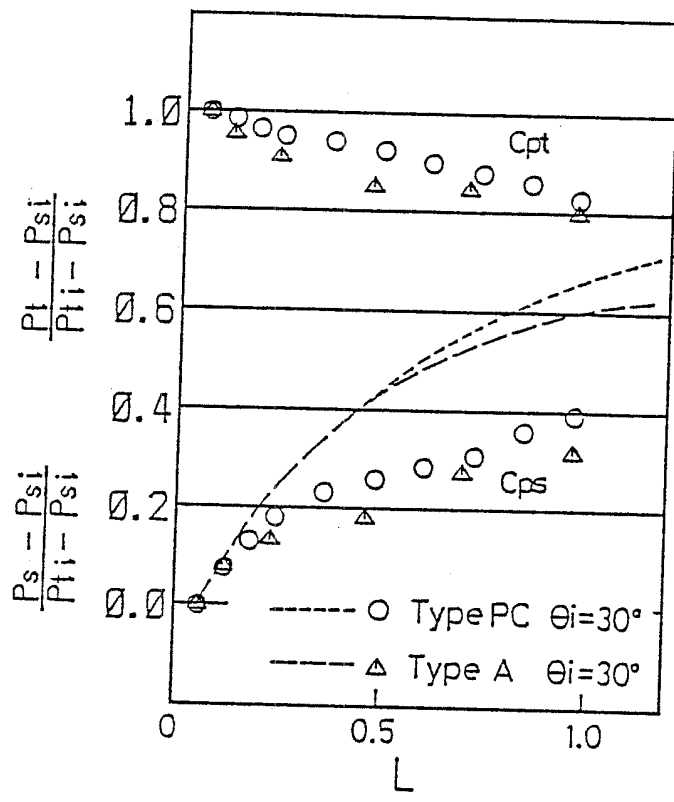

Fig. 11 Comparison of total pressure drop and pressure recovery between Type PC diffuser and Type A diffuser

Fig.12 shows a drop in the total pressure and static pressure recovery of Type D. A comparison is made between curved diffuser Type D and purely conical diffuser with guide fences? In case of purely conical diffuser, inlet flow angle $\theta i$ was equal to 25 degrees. Exact comparison is impossible because they have different inlet flow angle. But from this figure it is seen that these guide fences rectify the skewed boundary layer which has developed near the inner wall of mixed-flow diffuser and are effective to give favorable inner flow and high performance.

In Table 2 experimental static pressure coefficients Cps, theoretical static

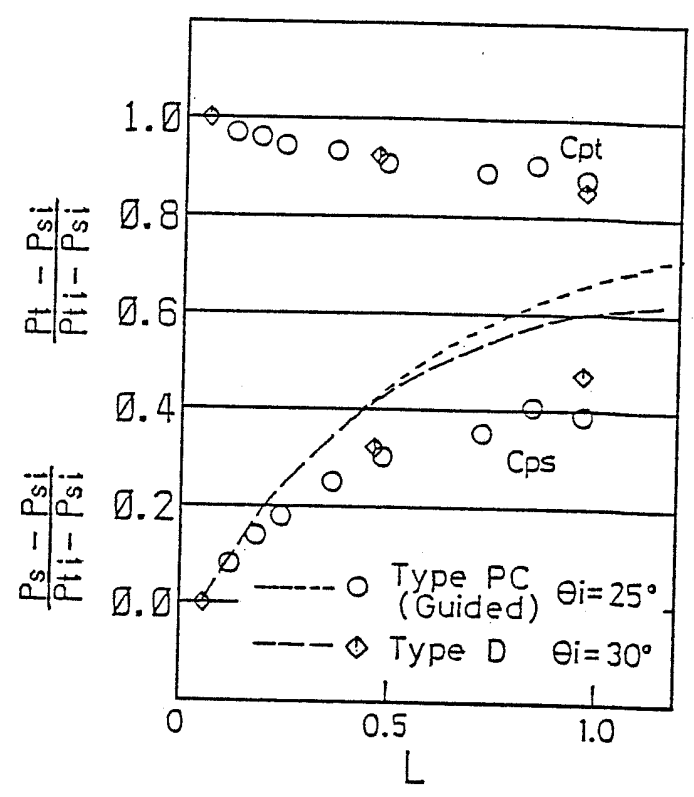

Fig. 12 Comparison of total pressure drop and pressure recovery between Type PC diffuser with guide fence and Type $D$ diffuser

pressure coefficients Cpsth and effectiveness of the diffuser $\eta_{\mathrm{p}}$ which is the ratio of Cps to Cpsth between the site No.1 and No. 6 are shown. Also, these values at 1.8 of radius ratio in purely conical diffuser are shown for the purpose of comparison. From this table it is seen that Type $D$ diffuser showes the most favorable performance.

\section{Conclusions}

(1) In case of axially curved mixed-flow diffuser the static pressure gradient normal to the diffuser walls due to the body force is considerable. With the radical development of a boundary layer near the inner wall, a low energy region begins to form near the inner wall. For this reason flow reversal is liable to occur near the inner wall unlike in purely conical diffuser.

(2) It is possible to prevent the development of a boundary layer and flow reversal by reducing the diffusion ratio of the diffuser or by shortening the length of streamlines in the diffuser owing to the large inlet flow angle. 
(3) Favorable flow and high performance are obtained even in the passage having high rate of diffusion by installing guide fences on the inner wall surface. In this study, the height of guide fences is about $1 / 5$ of diffuser width.

Acknowledgement

A part of this research was carried out with Grant-in-Aid for Scientific Research(C57550111) from the Ministry of Education of Japan.
The authors are indebted to $\mathrm{Mr}$. H.Matsunaga for his assistance in the

\section{References}

1 Wallace,F.J.,Atkey, R, and Whitfield, A., J.Mech.Eng.Sci.,17-6 (1975), 348. 2 Sakai,T.,Sanbe,M., and Nakayama, T. Trans.ASME, J.Eng. Power.,101-2 (1979)',
281 . 